\title{
Analyzing the Performance of Greedy Maximal Scheduling via Local Pooling and Graph Theory
}

\author{
Berk Birand, Maria Chudnovsky, Bernard Ries, Paul Seymour, Gil Zussman, and Yori Zwols
}

\begin{abstract}
Efficient operation of wireless networks and switches requires using simple (and in some cases distributed) scheduling algorithms. In general, simple greedy algorithms (known as Greedy Maximal Scheduling - GMS) are guaranteed to achieve only a fraction of the maximum possible throughput (e.g., $50 \%$ throughput in switches). However, it was recently shown that in networks in which the Local Pooling conditions are satisfied, GMS achieves $100 \%$ throughput. Moreover, in networks in which the $\sigma$-Local Pooling conditions hold, GMS achieves $\sigma \%$ throughput. In this paper, we focus on identifying the specific network topologies that satisfy these conditions. In particular, we provide the first characterization of all the network graphs in which Local Pooling holds under primary interference constraints (in these networks GMS achieves $100 \%$ throughput). This leads to a linear time algorithm for identifying Local Pooling-satisfying graphs. Moreover, by using similar graph theoretical methods, we show that in all bipartite graphs (i.e., input-queued switches) of size up to $7 \times n$, GMS is guaranteed to achieve $66 \%$ throughput, thereby improving upon the previously known $50 \%$ lower bound. Finally, we study the performance of GMS in interference graphs and show that in certain specific topologies its performance could be very bad. Overall, the paper demonstrates that using graph theoretical techniques can significantly contribute to our understanding of greedy scheduling algorithms.
\end{abstract}

Index Terms-Local pooling, scheduling, throughput maximization, graph theory, wireless networks, switches.

\section{INTRODUCTION}

The effective operation of wireless and wireline networks relies on the proper solution of the packet scheduling problem. In wireless networks, the main challenge stems from the need for a decentralized solution to a centralized problem. Even when centralized processing is possible, as is the case in inputqueued switches, designing low complexity algorithms that will enable efficient operation is a major challenge.

A centralized joint routing and scheduling policy that achieves the maximum attainable throughput region was presented by Tassiulas and Ephremides [28]. That policy applies to a multihop network with a stochastic packet arrival process and is guaranteed to stabilize the network whenever the arrival rates are within the stability region (i.e., it provides $100 \%$

B. Birand and G. Zussman are with the Department of Electrical Engineering, Columbia University, New York, NY (e-mail:\{berk, gil\}@ee.columbia.edu). M. Chudnovsky and Y. Zwols are with the Department of Industrial Engineering and Operations Research, Columbia University, New York, NY (e-mail:\{mchudnov,yz2198\}@ columbia.edu). B. Ries is with the Centre for Discrete Mathematics and its Applications (DIMAP), Warwick Business School, University of Warwick, Coventry, UK (e-mail: bernard.ries@wbs.ac.uk). P. Seymour is with the Department of Mathematics, Princeton University, Princeton, NJ (email:pds@math.princeton.edu). This work was partially supported by NSF grants DMS-0758364 and CNS0916263, CIAN NSF ERC under grant EEC-0812072, FNR grant TR-PDR BFR08-17, and ONR grant N00014-01-1-0608. throughput). The results of [28] have been extended to various settings of wireless networks and input-queued switches (e.g., [1], [10], [23]). However, algorithms based on [28] require the repeated solution of a global optimization problem, taking into account the queue backlog of every link. For example, even under simple primary interference constraints ${ }^{1}$, a maximum weight matching problem has to be solved in every slot, requiring an $O\left(n^{3}\right)$ algorithm.

Hence, there has been an increasing interest in simple (potentially distributed) algorithms. One such algorithm is the Greedy Maximal Scheduling (GMS) algorithm (also termed Maximal Weight Scheduling or Longest Queue First - LQF). This algorithm selects the set of served links greedily according to the queue lengths [13], [21]. Namely, at each step, the algorithm selects the heaviest link (i.e., with longest queue size), and removes it and the links with which it interferes from the list of candidate links. The algorithm terminates when there are no more candidate links. Such an algorithm can be implemented in a distributed manner [13], [18], [19].

It was shown that the GMS algorithm is guaranteed to achieve 50\% throughput in switches [8] and in general graphs under primary interference constraints [21]. It also was proved in [5], [26], [30] that under secondary interference constraints ${ }^{2}$ the throughput obtained by GMS may be significantly lower than the throughput under a centralized scheduler.

Although in arbitrary topologies the worst case performance of GMS can be very low, there are some topologies in which $100 \%$ throughput is achieved. Particularly, Dimakis and Walrand [9] presented sufficient conditions for GMS to provide $100 \%$ throughput. These conditions are referred to as Local Pooling (LoP) and are related to the structure of the network. Based on these conditions, it was shown that GMS achieves maximum throughput in tree network graphs under $k$-hop interference (for any $k$ ) [17], [31], in $2 \times n$ switches [4], and in a number of interference graph classes [31].

The LoP conditions were recently generalized to provide the $\sigma$-Local Pooling ( $\sigma$-LoP) conditions under which GMS achieves $\sigma \%$ throughput [16], [17] (the conditions were reformulated in [20]). Using these conditions, lower bounds on the guaranteed throughput in geometric graphs [17] and in graphs under secondary interference constraints [19] were obtained.

\footnotetext{
${ }^{1}$ Primary interference constraints imply that each pair of simultaneously active links must be separated by at least one hop (i.e., the set of active links at any point of time constitutes a matching).

${ }^{2}$ Secondary interference constraints imply that each pair of simultaneously active links must be separated by at least two hops (links). These constraints are usually used to model IEEE 802.11 networks [5].
} 
From a practical point of view, identifying graphs that satisfy LoP and $\sigma$-LoP can provide important building blocks for partitioning a network (e.g., via channel allocation) into subnetworks in which GMS performs well [4]. Another possible application is to add artificial interference constraints to a graph that does not satisfy the LoP conditions in order to turn it into a LoP-satisfying graph. Adding such constraints may decrease the stability region but would enable GMS to achieve a large portion of the new stability region.

While it is known that under primary interference some graph families (mainly trees and $2 \times n$ bipartite graphs) satisfy LoP, the exact structure of networks that satisfy LoP was not characterized. In this paper, we use graph theoretic methods to obtain the structure of all the network graphs that satisfy LoP under primary interference constraints (in these networks GMS achieves $100 \%$ throughput). This allows us to develop an algorithm that checks if a network graph satisfies LoP in time linear in the number of vertices, significantly improving over any other known method. We note that although primary interference constraints may not hold in many wireless networking technologies, the characterization provides an important theoretical understanding regarding the performance of simple greedy algorithms. It also shows that the $2 \times n$ switch is the largest switch for which $100 \%$ throughput is guaranteed.

We then focus on graphs in which GMS does not achieve $100 \%$ throughout. We consider bipartite network graphs (i.e., input-queued switches) and show that for bipartite graphs of size $k \times n$, where $k \leq 7$ and $n$ is arbitrary, GMS achieves at least 66\% throughput. Namely, for switches with up to 7 inputs or 7 outputs, the throughput under GMS is bounded from below by $66 \%$. This significantly improves upon the well known 50\% lower bound [8] and confirms many simulation studies (e.g., [11]) in which it was shown that greedy algorithms perform relatively well in switches. To show that this result does not extend to all bipartite graphs, we show that there exists a $10 \times 10$ bipartite graph for which $\sigma=0.6$.

Finally, we consider interference graphs ${ }^{3}$ and categorize different graph families according to their $\sigma$ values. In particular, we show that all co-strongly perfect graphs satisfy LoP. This class encapsulates all the classes of perfect LoP-satisfying interference graphs that were identified before (i.e., chordal graphs, interference graphs of trees, etc.). The observation increases the number of graphs known to satisfy LoP by an order of magnitude. Regarding $\sigma$-LoP we show that there are graphs with arbitrarily low $\sigma$. Since the worst case specific graph identified up to now had $\sigma=0.6$ [16] and the lowest lower bound known for a graph family was 1/6 [17], [19], this provides an important insight regarding graphs in which GMS may have bad performance. We conclude with briefly describing a simulation study that compares the performance of GMS to the optimal algorithm in graphs with low $\sigma$.

To conclude, the main contributions of this paper are two-

\footnotetext{
${ }^{3}$ Although it has been recently shown that in some cases the interference graph does not fully capture the wireless interference characteristics [24], it still provides a reasonable abstraction. Extending the results to general SINRbased constraints is a subject for further research.
}

fold: (i) a characterization of all network graphs in which Local Pooling holds under primary interference constraints (in these network graphs Greedy Maximal Scheduling is guaranteed to achieve $100 \%$ throughput) and (ii) improved lower bounds on the throughput performance of Greedy Maximal Scheduling in small switches. Overall, the paper demonstrates that using graph theoretical techniques can significantly contribute to our understanding of greedy scheduling algorithms.

This paper is organized as follows. In Section II we present the model. We characterize all graphs that satisfy LoP under primary interference constraints in Section III. In Section IV we show that GMS achieves $66 \%$ throughput in switches with up to 7 inputs. We study the performance of GMS in interference graphs in Section V and we conclude and discuss open problems in Section VI. Due to space constraints, some of the proofs are omitted and can be found in [2].

\section{MOdEL AND PRELIMINARIES}

In this section, we first present the network model under primary interference and then extend it for general interference. We also provide some graph theoretic definitions and derive results for graphs that exhibit certain symmetry.

\section{A. Network Graphs}

Consider a network graph $G=(V, E)$, where $V=$ $\{1, \ldots, n\}$ is the set of nodes, and $E \subseteq\{i j: i, j \in V, i \neq j\}$ is a set of links indicating pairs of nodes between which data flow can occur. Following the model of [4], [9], [16], [28], assume that time is slotted and that packets are of equal size, each packet requiring one time slot of service across a link. The model considers only single-hop traffic. A queue is associated with each edge in the network. We assume that the stochastic arrivals to edge $i j$ have long term rates $\lambda_{i j}$ and are independent of each other. We denote by $\vec{\lambda}$ the vector of the arrival rates $\lambda_{i j}$ for every edge $i j$. For more details regarding the queue evolution process under this model, see [4], [9], [16].

For a graph $G$, let $\mathbf{M}(G)$ be a $0-1$ matrix with $|E|$ rows, whose columns represent the maximal matchings of $G$. A scheduling algorithm selects a set of edges to activate at each time slot and transmits packets on those edges. Since they must not interfere under primary interference constraints, the selected edges form a matching. In other words, the scheduling algorithm picks a column $\boldsymbol{\pi}(t)$ from the maximal matching matrix $\mathbf{M}(G)$ at every time slot $t$. If $\boldsymbol{\pi}_{k}(t)=1$, one of the two nodes along edge $e_{k}$ can transmit, and the associated queue is decreased by one. We define the stability region (or capacity region) of a network as follows.

Definition 2.1 (Stability region [28]): The stability region of a network $G$ is defined by

$$
\Lambda^{*}=\{\vec{\lambda} \mid \vec{\lambda}<\vec{u} \text { for some } \vec{u} \in \operatorname{Co}(\mathbf{M}(G)),\},
$$

where $\operatorname{Co}(\mathbf{M}(G))$ is the convex hull of the columns of $\mathbf{M}(G)$ (inequality operators are taken element-wise when their operands are vectors).

A stable scheduling algorithm (which we also refer to as a throughput-optimal algorithm or an algorithm that achieves 
$100 \%$ throughput) is defined as an algorithm for which the Markov chain that represents the evolution of the queues is positive recurrent for all arrivals $\vec{\lambda} \in \Lambda^{*}$. It was shown in [28] that the Maximum Weight Matching algorithm that selects the matching with the largest total queue sizes at each slot is stable. When an algorithm is not throughput-optimal, the efficiency ratio $\gamma^{*}$ indicates the fraction of the stability region for which the algorithm is stable (in simple words, the queues are bounded for all arrival rates $\vec{\lambda} \in \gamma^{*} \Lambda^{*}$ ).

We briefly reproduce the definitions of Local Pooling (LoP) presented in [4], [9]. ${ }^{4}$ In the following, e denotes the vector having each entry equal to one.

Definition 2.2 (Subgraph Local Pooling - SLoP): A network graph $G$ satisfies SLoP, if there exists $\boldsymbol{\alpha} \in[0,1]^{|E|}$ such that $\boldsymbol{\alpha}^{T} \mathbf{M}(G)=\mathbf{e}^{T}$.

This definition also corresponds to associating a weight, denoted $\boldsymbol{\alpha}(e)$, to all edges $e \in E$, such that

$$
\sum_{e \in Z} \boldsymbol{\alpha}(e)=1 \text { for every maximal matching } Z \text { in } G \text {. }
$$

If a vector $\boldsymbol{\alpha}$ satisfies the above condition, we will say that it is a good edge weighting.

Definition 2.3 (Overall Local Pooling - OLoP): $A$ network graph $G$ satisfies OLoP, if every subgraph $S$ of $G$ satisfies SLoP.

In [9], Dimakis and Walrand proved that if a graph satisfies OLoP, GMS achieves $100 \%$ throughput. In networks in which OLoP is not satisfied, $\sigma$-Local Pooling [16], [17] provides a way of estimating the efficiency ratio $\gamma^{*}$ of GMS. Below, we provide a different definition called $\sigma$-SLoP that is equivalent to the original one from [16], [17].

Definition 2.4 ( $\sigma$-SLoP - Xi et. al. [20]): A network graph $G$ satisfies $\sigma-S L o P$, if and only if there exists a vector $\boldsymbol{\alpha} \in[0,1]^{|E|}$ such that

$$
\sigma \mathbf{e}^{T} \leq \boldsymbol{\alpha}^{T} \mathbf{M}(G) \leq \mathbf{e}^{T} .
$$

This definition can also be expressed as a Linear Program [20].

Clearly, if a graph satisfies $\sigma$-SLoP, it also satisfies $\sigma^{\prime}$-SLoP for every $\sigma^{\prime}<\sigma$. Therefore, it is sufficient to focus on the largest value of $\sigma$ such that $G$ satisfies $\sigma$-SLoP. This value is denoted by $\sigma(G)$ :

$$
\sigma(G):=\max \{\sigma \mid G \text { satisfies } \sigma-\mathrm{SLoP}\} .
$$

We say that a graph satisfies $\sigma$-OLoP, if all of its subgraphs satisfy $\sigma$-SLoP. We can then define the local pooling factor of a graph as follows:

Definition 2.5 (Joo et. al. [16]): The local pooling factor $\sigma^{*}(G)$ of a network graph $G$ is the largest value of $\sigma$ for which $\sigma$-SLoP is satisfied for all subgraphs $S$.

This definition can also be written in terms of $\sigma(S)$ :

$$
\sigma^{*}(G):=\min \{\sigma(S) \mid \text { for all subgraphs } S \text { of } G\} .
$$

It was proved in [16] that the local pooling factor $\sigma^{*}$ of a graph is equal to the efficiency ratio $\gamma^{*}$ of GMS in that

\footnotetext{
${ }^{4}$ This definition slightly differs from that in [4] by setting the sum equal to $\mathbf{e}^{T}$ instead of $c \mathbf{e}^{T}$, where $c$ is a positive constant.
}

graph. For instance, if a graph has a local-pooling factor of $2 / 3$, GMS is stable for all arrival rates $\vec{\lambda} \in \frac{2}{3} \Lambda^{*}$ and therefore achieves $66 \%$ throughput. Note that $\sigma^{*}(G)=1$, if and only if $G$ satisfies the OLoP condition.

\section{B. Interference Graphs}

We now generalize the model by introducing interference graphs. Based on the network graph and the interference constraints, the interference between network links can be modeled by an interference graph (or a conflict graph) $G_{I}=\left(V_{I}, E_{I}\right)$ [15]. We assign $V_{I}=E$. Thus, each edge $e_{k}$ in the network graph is represented by a node $v_{k}$ in the interference graph, and an edge $v_{i} v_{j}$ in the interference graph indicates a conflict between network graph links $e_{i}$ and $e_{j}$ (i.e., transmissions on $e_{i}$ and $e_{j}$ cannot take place simultaneously). Under primary interference, the interference graph $G_{I}$ corresponds to the line graph of $G$.

The model and the LoP theory described so far extend to interference graphs. The nodes of $G_{I}$ correspond to queues to which packets arrive according to a stochastic process at every time slot. A scheduling algorithm must pick an independent set at each slot so that neighboring nodes will not be activated simultaneously. Each column of the matrix $\mathbf{M}\left(G_{I}\right)$ corresponds to a maximal independent set of $G_{I}$. An algorithm which selects the independent set with the largest weights (i.e., solves the Maximum Weight Independent Set Problem) is stable. SLoP corresponds to finding a vector $\boldsymbol{\alpha} \in[0,1]^{\left|V_{I}\right|}$ that assigns a weight $\boldsymbol{\alpha}(u)$ to each node $u$ such that $\sum_{u \in I} \boldsymbol{\alpha}(u)=1$ for every maximal independent set $I$ in $G_{I}$. If such a vector exists, we will call it a good node weighting. For OLoP to be satisfied, SLoP must be satisfied by all induced subgraphs (i.e., with respect to node removals). $\sigma$-SLoP and $\sigma$-OLoP extend to this case in a very similar way.

\section{Graph Theoretic Definitions}

We briefly review some definitions from graph theory that are required in the following sections (for details, see [29]). For a graph $G$, we denote by $N(v)$ the set of neighbors of $v$ and by $\operatorname{deg}(v)=|N(v)|$ the degree of $v$. For $x, y \in V(G)$, we say that $x$ is a clone of $y$ if $N(x)=N(y)$. We say that $X \subseteq V(G)$ is a clique (independent set) if the vertices in $X$ are pairwise adjacent (non-adjacent). A matching $M$ is said to cover a vertex $v$, if there exists an edge in $M$ that is incident with $v$. For $x \in V(G)$, we denote by $G-x$ the graph obtained from $G$ by deleting $x$ and all edges incident with it. An induced subgraph of $G$ is a subgraph of $G$ that can be obtained from $G$ by repeatedly deleting a vertex and all edges incident with it. For two graphs $G_{1}, G_{2}$, an isomorphism from $G_{1}$ to $G_{2}$ is a bijection $\phi: V\left(G_{1}\right) \rightarrow V\left(G_{2}\right)$ such that $u v \in E\left(G_{1}\right)$, if and only if $\phi(u) \phi(v) \in E\left(G_{2}\right)$. Two graphs $G_{1}, G_{2}$ are isomorphic, if there exists an isomorphism from $G_{1}$ to $G_{2}$. An automorphism of $G$ is an isomorphism from $G$ to itself. A graph $G$ is edge-transitive, if for all $u v, w x \in E(G)$, there exists an automorphism $\phi$ of $G$ such that $\phi(u) \phi(v)=w x$. A graph $G$ is vertex-transitive, if for all $u, v \in V(G)$, there exists an automorphism $\phi$ of $G$ such that $\phi(u)=v$. A connected 
component of $G$ is a maximal connected induced subgraph of $G$. Finally, for $n \geq 1$, we let $K_{n}$ denote the complete graph on $n$ nodes and, for $t \geq 1$, we let $K_{t, n}$ denote the $t \times n$ complete bipartite graph.

\section{D. $\sigma(G)$-values and Bounds on $\sigma(G)$}

We now describe a simple method to compute a lower bounds on $\sigma(G)$ and provide a method for calculating $\sigma(G)$ of edge- and vertex-transitive graphs. These are graphs that exhibit a high degree of symmetry (e.g., cycles). We will use the following notation:

$$
\begin{aligned}
& \nu(G)=\max \{|Z|: Z \text { is a maximal matching in } G\}, \\
& \mu(G)=\min \{|Z|: Z \text { is a maximal matching in } G\} .
\end{aligned}
$$

The following lemma presents a lower-bound on $\sigma(G)$ [19].

Lemma 2.1 (Leconte et al. [19]): For any graph $G$, $\sigma(G) \geq \mu(G) / \nu(G)$.

Using Definition 2.4, we provide an alternative proof to this lemma in [2]. To demonstrate the benefits of the $\sigma$-OLoP definition, we provide a very simple proof to the fact that GMS achieves $50 \%$ throughput in any network graph $G$ (shown in different methods in [8], [21]). First, note that the size of any maximal matching is at least half the size of a maximum matching [25], which means that $\mu(G) \geq \nu(G) / 2$, for all $G$. By Lemma 2.1 and (2), it follows that $\sigma^{*}(G) \geq 1 / 2$ for every graph $G$, and therefore that $\gamma^{*} \geq 1 / 2$.

For edge-transitive graphs, the following lemma is a stronger counterpart of Lemma 2.1 (the proof is in [2]):

Lemma 2.2: If $G$ is edge-transitive, then $\sigma(G)=$ $\mu(G) / \nu(G)$.

The lemmas introduced previously also extend to general interference graphs. We define the independent set counterparts of $\mu(G)$ and $\nu(G)$ as follows:

$$
\bar{\nu}(G)=\max \{|S|: Z \text { is a maximal independent set in } G\},
$$$$
\bar{\mu}(G)=\min \{|S|: Z \text { is a maximal independent set in } G\} .
$$

It is easy to generalize Lemma 2.1 to interference graphs $G$ and show that $\sigma(G) \geq \bar{\mu}(G) / \bar{\nu}(G)$. For vertex-transitive graphs, the following holds:

Lemma 2.3: If $G$ is vertex-transitive, then $\sigma(G)=$ $\bar{\mu}(G) / \bar{\nu}(G)$.

\section{NETWORK GRAPHS THAT SATISFY OLOP UNDER PRIMARY INTERFERENCE}

Only a small collection of network graphs have been shown to satisfy $\mathrm{OLoP}$ under primary interference. Among the known cases are trees [4], [17], and $2 \times n$ bipartite graphs [4]. The main result of this section is a description of the structure of all network graphs that satisfy OLoP under primary interference. This structure shows that such graphs are relatively easy to construct and, moreover, they can be recognized in linear time.

Define the following families of graphs. For $k \geq 3$, let $C_{k}$ be a cycle with $k$ edges (or, equivalently, $k$ nodes). For $k \geq 0$ and $p, q \in\{5,7\}$, let $D_{k}^{p, q}$ be the graph formed by the union of two cycles of size $p$ and $q$ joined by a $k$-edge

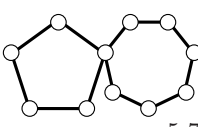

(a) $D_{0}^{5,7}$ (b) $D_{3}^{5,5}$

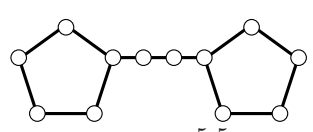

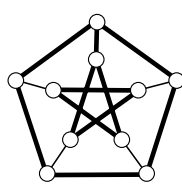

(c) Petersen graph
Fig. 1. Graphs (a) and (b): examples of graphs from the family $D_{k}^{p, q}$, all of which fail OLoP under primary interference. Graph (c): the Petersen graph. This graph does not satisfy OLoP because it contains, among other graphs, $C_{6}$ and $D_{1}^{5,5}$ (bold edges) as subgraphs.

path (where $k \geq 0$ ). If $k=0$, the cycles share a common node (see Fig. 1-(a) and 1-(b)). Let $\mathcal{F}=\left\{C_{k} \mid k \geq 6, k \neq\right.$ $7\} \cup\left\{D_{k}^{p, q} \mid k \geq 0 ; p, q \in\{5,7\}\right\}$. For two graphs $G$ and $H$, we say that $G$ contains $H$ as a subgraph if $G$ has a subgraph that is isomorphic to $H$. We will say that a graph $G$ is $\mathcal{F}$-free, if it does not contain any graph $F \in \mathcal{F}$ as a subgraph.

We will focus on connected graphs, because it is easy to see that a graph satisfies OLoP, if and only if all its connected components satisfy OLoP. So we may assume without loss of generality that all graphs in this section are connected graphs.

The results in this section are three-fold. First, in Subsection III-A, we give a structural description of all $\mathcal{F}$-free graphs. Second, in Subsection III-B, we will use this description to prove the following theorem:

Theorem 3.1: A network graph $G$ satisfies OLoP under primary interference, if and only if $G$ is $\mathcal{F}$-free.

Theorem 3.1 shows that if a network graph $G$ does not satisfy OLOP under primary interference, then $G$ contains some $F \in \mathcal{F}$ as a subgraph. For example, it was previously shown that the Petersen graph (Fig. 1-(c)) fails OLoP [16]. Using Theorem 3.1 we can immediately see this from the fact that it contains, for example, $C_{6}$ and $D_{1}^{5,5}$ as a subgraph.

Testing whether a network graph satisfies SLoP previously required enumerating all maximal matchings (of which there are an exponential number) and solving a Linear Program [9]. To test the OLoP condition, this procedure had to be repeated for every subgraph. The weakness of this approach is its large computational effort. In Subsection III-C, we present the third result, which uses the structure of $\mathcal{F}$-free graphs to construct an algorithm that decides in linear time whether a graph satisfies OLoP, as described in the following theorem:

Theorem 3.2: It can be decided in $O(|V(G)|)$ time whether a network graph $G$ satisfies OLoP under primary interference.

\section{A. The Structure of $\mathcal{F}$-free Graphs}

We will start with a structural description of $\mathcal{F}$-free graphs. The reason for our interest in $\mathcal{F}$-free graphs is the fact (which will be proved in Subsection III-B) that the class of $\mathcal{F}$-free graphs is precisely the class of network graphs that satisfy OLoP under primary interference.

We will describe the structure of $\mathcal{F}$-free graphs in terms of the so-called 'block decomposition'. Let $G$ be a connected graph. We call $x \in V(G)$ a cut-node of $G$, if $G-x$ is not connected. We call a maximal connected induced subgraph $B$ of $G$ such that $B$ has no cut-node a block of $G$. Let $B_{1}, B_{2}, \ldots, B_{q}$ 
be the blocks of $G$. We call the collection $\left\{B_{1}, B_{2}, \ldots, B_{q}\right\}$ the block decomposition of $G$. It is known that the block decomposition is unique and that $E\left(B_{1}\right), E\left(B_{2}\right), \ldots, E\left(B_{q}\right)$ forms a partition of $E(G)$ (e.g., [29]). Furthermore, the node sets of every two blocks intersect in at most one node and this node is a cut-node of $G$.

Block decompositions give a tree-like decomposition of a graph in the following sense. Construct the block-cutpoint graph of $G$ by keeping the cut-nodes of $G$ and replacing each block $B_{i}$ of $G$ by a node $b_{i}$. Make each cut-node $v$ adjacent to $b_{i}$ if and only if $v \in V\left(B_{i}\right)$. It is known that the blockcutpoint graph of $G$ forms a tree (e.g., [29]). With this tree-like structure in mind, we say that a block $B_{i}$ is a leaf block if it contains at most one cut-node of $G$. Clearly, if $q \geq 2$, then $\left\{B_{i}\right\}_{i=1}^{q}$ contains at least two leaf blocks.

It turns out that the block decomposition of an $\mathcal{F}$-free graph is relatively simple in the sense that there are only two types of blocks. The types are defined by the following two families of graphs. Examples of these families appear in Fig. 2.

$\mathcal{B}_{1}$ : Construct $\mathcal{B}_{1}$ as follows. Let $H$ be a graph with $V(H)=$ $\left\{c_{1}, c_{2}, \ldots, c_{k}\right\}$, with $k \in\{5,7\}$, such that

1) $c_{1}-c_{2}-\cdots-c_{k}-c_{1}$ is a cycle;

2) if $k=5$, then the other adjacencies are arbitrary; if $k=7$, then all other pairs are non-adjacent, except possibly $\left\{c_{1}, c_{4}\right\},\left\{c_{1}, c_{5}\right\}$ and $\left\{c_{4}, c_{7}\right\}$.

Then, $H \in \mathcal{B}_{1}$.

Now iteratively perform the following operation. Let $H^{\prime} \in \mathcal{B}_{1}$ and let $x \in V\left(H^{\prime}\right)$ with $\operatorname{deg}(x)=2$. Construct $H^{\prime \prime}$ from $H^{\prime}$ by adding a node $x^{\prime}$ such that $N\left(x^{\prime}\right)=N(x)$. Then, $H^{\prime \prime} \in \mathcal{B}_{1}$. We say that a graph is of the $\mathcal{B}_{1}$ type if it is isomorphic to a graph in $\mathcal{B}_{1}$.

$\mathcal{B}_{2}$ : Let $\mathcal{B}_{2}=\left\{K_{2}, K_{3}, K_{4}\right\} \cup\left\{K_{2, t}, K_{2, t}^{+} \mid t \geq 2\right\}$, where $K_{2, t}^{+}$is constructed from $K_{2, t}$ by adding an edge between the two nodes on the side that has cardinality 2 . We say that a graph is of the $\mathcal{B}_{2}$ type, if it is isomorphic to a graph in $\mathcal{B}_{2}$.

In simple words, graphs of the $\mathcal{B}_{1}$ type are constructed by starting with a cycle of length five or seven. Then we may add some additional edges between nodes of the cycle, subject to some constraints. Finally, we may iteratively take a node $x$ of degree 2 and add a clone $x^{\prime}$ of $x$. It will turn out that $\mathcal{F}$-free graphs have at most one block of the $\mathcal{B}_{1}$ type and that all other blocks are of the $\mathcal{B}_{2}$ type. This means that $\mathcal{F}$-free graphs can be constructed by starting with a block that is either of the $\mathcal{B}_{1}$ or of the $\mathcal{B}_{2}$ type, and then iteratively adding a block of the $\mathcal{B}_{2}$ type by 'glueing' it on an arbitrary node.

Fig. 2 shows an example of an $\mathcal{F}$-free graph. The tree-like structure is clearly visible. The graph has one block of the $\mathcal{B}_{1}$ type with $k=7$. This block consists of a cycle of length 7 together with two clones. The other blocks are of the $\mathcal{B}_{2}$ type. Some of them are attached to the block of the $\mathcal{B}_{1}$ type through a cut-node. Others are attached to other blocks of the $\mathcal{B}_{1}$ type. Notice that trees and $2 \times n$ complete bipartite graphs, which were previously known to satisfy OLoP [4], [17], are, as should be expected, subsumed by this structure.

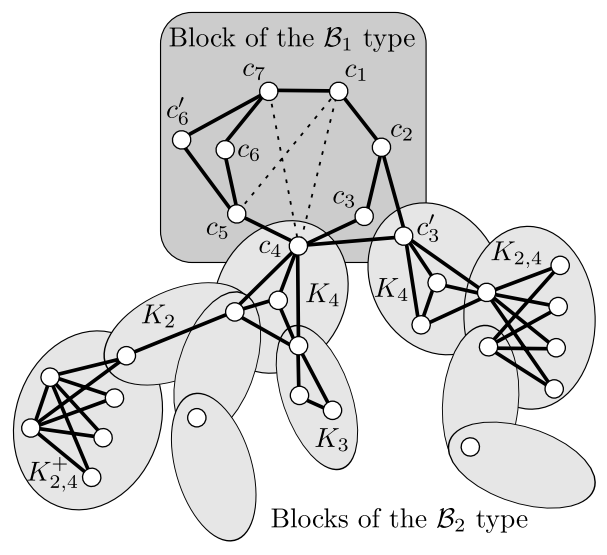

Fig. 2. An example of an $\mathcal{F}$-free graph (the dashed edges may or may not be present). The ellipses show the blocks of the graph.

The goal of this subsection is to prove the following formal version of the characterization given above:

Theorem 3.3: Let $G$ be a connected graph and let $\left\{B_{1}, B_{2}, \ldots, B_{q}\right\}$ be the block decomposition of $G$. Then $G$ is $\mathcal{F}$-free, if and only if there is at most one block that is of the $\mathcal{B}_{1}$ type and all other blocks are of the $\mathcal{B}_{2}$ type.

The proof of the 'if' direction is straightforward. Here, we will give a proof sketch of the 'only-if' direction in a number of steps. For a block $B$ in an $\mathcal{F}$-free graph, its type depends on the size of the longest cycle in $B$. It will turn out that if $B$ contains a cycle of length 5 or 7 , then $B$ is of the $\mathcal{B}_{1}$ type. Otherwise, $B$ is of the $\mathcal{B}_{2}$ type. We have the following result on blocks that have a cycle of length five or seven.

Lemma 3.1: Let $G$ be an $\mathcal{F}$-free graph and let $B$ be a block of $G$. Let $F$ be a cycle in $B$ that has maximum length. If $|V(F)| \geq 5$, then $B$ is of the $\mathcal{B}_{1}$ type.

Next, we deal with blocks that do not contain a cycle of length 5 or 7. It follows from the definition of $\mathcal{F}$-free graphs that such blocks do not have cycles of length at least 5 . Maffray [22] proved the following theorem:

Theorem 3.4: [Maffray [22]] Let $G$ be a graph. Then, the following statements are equivalent:

(1) $G$ does not contain any odd cycle of length at least 5 .

(2) For every connected subgraph $G^{\prime}$ of $G$, either $G^{\prime}$ is isomorphic to $K_{4}$, or $G^{\prime}$ is a bipartite graph, or $G^{\prime}$ is isomorphic to $K_{2, t}^{+}$for some $t \geq 1$, or $G^{\prime}$ has a cut-node.

Theorem 3.4 implies the following lemma.

Lemma 3.2: Let $G$ be an $\mathcal{F}$-free graph and let $B$ be a block of $G$. Suppose that $B$ contains no cycle of length at least 5 . Then, $B$ is of the $\mathcal{B}_{2}$ type.

We use the previous lemmas to prove Theorem 3.3 in [2].

B. Network graphs satisfy OLoP under primary interference, if and only if they are $\mathcal{F}$-free

Now that we have described the structure of all $\mathcal{F}$-free graphs, we use this structure to prove Theorem 3.1 which states that a network graph satisfies OLoP under primary interference, if and only if it is $\mathcal{F}$-free. It was shown in [4] (Theorems 2 and 3) that all cycles of length $k \geq 6, k \neq 7$ fail 
SLoP. ${ }^{5}$ Therefore, such cycles do not appear as subgraphs in graphs that satisfy OLoP. The following lemma shows that the same is true for the graphs $D_{k}^{p, q}$.

Lemma 3.3: $D_{k}^{p, q}$ fails SLoP for all $p, q \in\{5,7\}, k \geq 0$.

The results from [4] together with Lemma 3.3 imply the following result:

Corollary 3.1: Graphs that satisfy OLoP are $\mathcal{F}$-free.

Corollary 3.1 settles the 'only-if' direction of Theorem 3.1. To prove the 'if' direction, we will start with a useful lemma. We will give the idea of the proof, the full proof being in [2].

Lemma 3.4: Let $G$ be a graph and $x, x^{\prime} \in V(G)$ such that $\operatorname{deg}(x)=2$ and $x^{\prime}$ is a clone of $x$. Then, $G$ satisfies SLoP.

Proof idea: Let $\left\{z_{1}, z_{2}\right\}=N(x)$. Define $\boldsymbol{\alpha} \in[0,1]^{|E|}$ by letting $\boldsymbol{\alpha}(e)=1 / 2$, if $e$ is incident with $z_{1}$ or $z_{2}$, and $e \neq z_{1} z_{2}, \boldsymbol{\alpha}\left(z_{1} z_{2}\right)=1$, if $z_{1} z_{2} \in E(G)$, and $\boldsymbol{\alpha}(e)=0$ for all other edges $e$. Now every maximal matching uses either two edges $e_{1}, e_{2}$ with $\boldsymbol{\alpha}\left(e_{1}\right)=\boldsymbol{\alpha}\left(e_{2}\right)=1 / 2$, or edge $z_{1} z_{2}$.

The following lemma is the crucial step in settling the "onlyif' direction of Theorem 3.1. Again, we give the proof idea.

Lemma 3.5: Every connected $\mathcal{F}$-free satisfies SLoP.

Proof idea: Let $G$ be a connected $\mathcal{F}$-free graph and let $\left\{B_{1}, B_{2}, \ldots, B_{q}\right\}$ be the block decomposition of $G$. It follows from Theorem 3.3 that there is at most one block $B_{i}$ that is of the $\mathcal{B}_{1}$ type and all other blocks are of the $\mathcal{B}_{2}$ type. We will construct a good edge weighting $\boldsymbol{\alpha}$ for $G$.

Suppose first that $G$ has a leaf block $B_{i}$ of the $\mathcal{B}_{2}$ type. If $q=2$, then let $x$ be the cut-node of $G$ in $V\left(B_{i}\right)$. If $q=1$, let $x \in V\left(B_{i}\right)$ be arbitrary. There are four cases:

(1) $B_{i}$ is isomorphic to $K_{2}$ : let $x, v$ denote the nodes of $B_{i}$. Let $\boldsymbol{\alpha}(e)=1$ for all edges incident with $x$ and $\boldsymbol{\alpha}(e)=0$ for every other edge $e$.

(2) $B_{i}$ is isomorphic to $K_{3}$ : let $\boldsymbol{\alpha}(e)=1$ for all $e \in E\left(B_{i}\right)$ and $\boldsymbol{\alpha}(e)=0$ for every other edge $e$.

(3) $B_{i}$ is isomorphic to $K_{4}$ : let $x, v_{1}, v_{2}, v_{3}$ denote the nodes of $B_{i}$ and let $\boldsymbol{\alpha}\left(v_{1} v_{2}\right)=\boldsymbol{\alpha}\left(v_{1} v_{3}\right)=\boldsymbol{\alpha}\left(v_{2} v_{3}\right)=1$ and $\boldsymbol{\alpha}(e)=$ 0 for all $e \in\left(E(G) \backslash\left\{v_{1} v_{2}, v_{1} v_{3}, v_{2} v_{3}\right\}\right)$.

(4) $B_{i}$ is isomorphic to $K_{2, t}$ or $K_{2, t}^{+}$for some $t \geq 2$ : let $V\left(B_{i}\right)=V_{1} \cup V_{2}$ such that $\left|V_{1}\right|=2$ and $V_{2}$ is an independent set. Let $V_{1}=\left\{y_{1}, y_{2}\right\}$ and let $V_{2}=\left\{z_{1}, z_{2}, \ldots, z_{t}\right\}$. If $B_{i}$ is isomorphic to $K_{2,2}^{+}$and $x \in V_{2}$, then assume that $x=z_{1}$ and set $\boldsymbol{\alpha}\left(y_{1} z_{2}\right)=\boldsymbol{\alpha}\left(y_{2} z_{2}\right)=\boldsymbol{\alpha}\left(y_{1} y_{2}\right)=1$. Otherwise, $B_{i}$ contains nodes $p, p^{\prime}$ such that $\operatorname{deg}(p)=\operatorname{deg}\left(p^{\prime}\right)=2$ and $p^{\prime}$ is a clone of $p$, and hence, the result follows from Lemma 3.4.

Thus, we may assume that $G$ does not have a leaf block of the $\mathcal{B}_{2}$ type. Since if $q \geq 2, G$ has at least two leaf blocks, and hence, at least one leaf block of the $\mathcal{B}_{2}$ type, we may assume that $q=1$ and $G=B_{1}$ is of the $\mathcal{B}_{1}$ type. Let $H, k$ be as in the definition of $\mathcal{B}_{1}$. It follows from the definition of $H$ that $|V(H)|=k$. First, suppose that $V(G) \backslash V(H) \neq \emptyset$. Then, it follows from the definition of $\mathcal{B}_{1}$ that there exist two nodes $x, x^{\prime}$ such that $\operatorname{deg}(x)=\operatorname{deg}\left(x^{\prime}\right)=2$ and $x^{\prime}$ is a clone of $x$. It follows from Lemma 3.4 that $G$ satisfies SLoP. So we may assume that $V(G)=V(H)$. If $k=5$, then every maximal

\footnotetext{
${ }^{5}$ Although the case considered in [4] pertains to interference graphs, the network case is identical since the interference graph (under primary interference) of a cycle is a cycle of the same length.
}

matching has size two, and hence, we may set $\boldsymbol{\alpha}(e)=1 / 2$ for all $e \in E(G)$. If $k=7$, then every maximal matching has size three, and hence, we may set $\boldsymbol{\alpha}(e)=1 / 3$ for all $e \in E(G)$.

We are now in a position to prove Theorem 3.1:

Proof of Theorem 3.1: Corollary 3.1 is the 'only-if' part of the theorem. For the 'if' part, since every subgraph of $G$ is $\mathcal{F}$-free, it follows from Lemma 3.5 that every subgraph of $G$ satisfies SLoP. Therefore, $G$ satisfies OLoP.

\section{Recognizing network graphs that satisfy OLoP under pri- mary interference}

Having described the structure of graphs that satisfy OLoP, we provide an efficient algorithm for testing whether a given network graph satisfies OLoP under primary interference. A useful observation is the following.

Lemma 3.6: $|E(G)| \leq 2|V(G)|$ for every $\mathcal{F}$-free graph $G$.

This puts us in a position to prove Theorem 3.2.

Proof idea of Theorem 3.2: We may assume that $G$ is connected. By Theorems 3.1 and 3.3, it suffices to check whether $G$ has the structure described in Theorem 3.3. We propose the following algorithm. Let $n=|V(G)|$ and $m=$ $|E(G)|$. First, check that $m \leq 2 n$, because otherwise $G$ is not $\mathcal{F}$-free by Lemma 3.6 and we can stop. Now, construct the block decomposition $\left\{B_{1}, B_{2}, \ldots, B_{q}\right\}$ of $G$. Since $m \leq 2 n$, this can be done in $O(n+m)=O(n)$ time (see e.g., [12]). For each block $B_{i}$, test in $O\left(\left|V\left(B_{i}\right)\right|\right)$ time whether $B_{i}$ is of the $\mathcal{B}_{2}$ type. If $G$ has more than one block that is not of the $\mathcal{B}_{2}$ type, then $G$ is not $\mathcal{F}$-free and we stop. If we encounter no such block, then $G$ is $\mathcal{F}$-free and we stop. Next, check whether $B^{*}$ is of the $\mathcal{B}_{1}$ type using multiple applications of Bodlaender's algorithm [3] which, for fixed $k$, finds a cycle of length at least $k$ in a given graph $H$, if it exists, in $O\left(k ! 2^{k}|V(H)|\right)$ time. Checking this can be done in $O(|V(B)|)$ time. Therefore, the overall complexity of the algorithm is $O(n)$.

\section{IV. $t \times n$ SWITCHES WITH $t \leq 7$ SATISFY $\sigma^{*} \geq 2 / 3$}

In the previous section, we characterized the full set of graphs that satisfy OLoP. It is only natural to ask the question: what happens to graphs that do not satisfy OLoP? In this section, we will show that every bipartite graph $G$ that has one side with at most 7 nodes satisfies $\sigma^{*}(G) \geq 2 / 3$, which implies that $\sigma^{*}\left(K_{t, n}\right)=2 / 3$ for $3 \leq t \leq 7, n \geq 3$. We also note that this bound is close to being tight by presenting a bipartite graph with 10 nodes on one side for which $\sigma^{*}(G)<2 / 3$. Consider the so-called Desargues graph $\mathcal{D}$ in Fig. 3. $\mathcal{D}$ is edge-transitive and hence it follows from Lemma 2.2 and the fact that $\nu(\mathcal{D})=10$ and $\mu(\mathcal{D})=6$ that $\sigma(\mathcal{D})=3 / 5$. Since $\mathcal{D}$ is a subgraph of $K_{10,10}$, this implies that $\sigma^{*}\left(K_{t, n}\right) \leq 3 / 5$ for all $t \geq 10, n \geq 10$.

We now concentrate on subgraphs of $K_{t, n}$ with $t \leq 7, n \geq$ 1 . We will start with some easy observations that help give a lower bound on $\sigma(G)$.

Lemma 4.1: Let $G$ be a graph.

(a) If there exists $v \in V(G)$ such that every maximal matching in $G$ covers $v$, then $\sigma(G)=1$. 


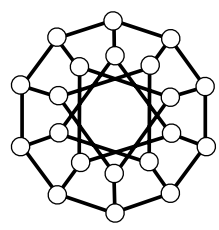

Fig. 3. The Desargues graph $\mathcal{D}$ for which $\sigma(\mathcal{D})=0.6$ and which is a subgraph of $K_{10,10}$, showing that $\sigma^{*}\left(K_{10,10}\right) \leq 0.6$.

(b) If $\operatorname{deg}(v)=1$ for some $v \in V(G)$, then $\sigma(G)=1$.

(c) If $\operatorname{deg}(v)=2$ for some $v \in V(G)$, then $\sigma(G) \geq 2 / 3$.

Proof: Part (a): let $\boldsymbol{\alpha}(e)=1$ for all edges incident with $v$ and $\boldsymbol{\alpha}(e)=0$ for all other edges. Clearly, every maximal matching $Z$ satisfies $\sum_{e \in Z} \boldsymbol{\alpha}(e)=1$. This proves (a). Part (b) follows immediately because if $\operatorname{deg}(v)=1$, then every maximal matching covers the unique neighbor $u$ of $v$. Part (c): let $a, b$ be the neighbors of $v$. Let $\boldsymbol{\alpha}(a v)=\boldsymbol{\alpha}(b v)=2 / 3$, $\boldsymbol{\alpha}(a b)=1$ if $a b \in E(G), \boldsymbol{\alpha}(e)=1 / 3$ for all edges $e \notin$ $\{a b, b v, a v\}$ that are incident with $a$ or $b$, and $\boldsymbol{\alpha}(e)=0$ for all other edges. It is not hard to see that $\sum_{e \in Z} \boldsymbol{\alpha}(e) \geq 2 / 3$ for every maximal matching $Z$ in $G$. This proves (c), thus proving Lemma 4.1.

By using the conditions given in Lemmas 4.1 and 2.1, we prove the following lemma in [2]:

Lemma 4.2: Let $G$ be a bipartite graph with $\mu(G) \leq 4$. Then $\sigma(G) \geq 2 / 3$.

Lemma 4.2 has the following corollaries:

Corollary 4.1: Every bipartite graph $G$ with $\nu(G) \leq 7$ satisfies $\sigma^{*}(G) \geq 2 / 3$.

It is already known that $\sigma^{*}\left(K_{t, n}\right)=1$ for $t \in\{1,2\}$. For $3 \leq t \leq 7$, we obtain:

Corollary 4.2: $\sigma^{*}\left(K_{t, n}\right)=2 / 3$ for all $3 \leq t \leq 7, n \geq 3$.

\section{INTERFERENCE GRAPHS AND THEIR $\sigma^{*}$-VALUES}

Our focus so far has been on network graphs and primary interference constraints. We now consider general interference graphs that represent various transmission constraints. Recall that under general interference constraints, a scheduling algorithm has to select an independent set from the interference graph at each slot. We are interested in the performance of a low-complexity GMS algorithm which greedily picks the nodes with the largest weight (this algorithm is also referred to as the Maximal Weighted Independent Set algorithm). The results are summarized in Fig. 4 which illustrates throughput guarantees of several graph families.

\section{A. OLoP-satisfying Interference Graphs}

We first show that the OLoP condition holds in a large subclass of perfect graphs which we will call co-strongly perfect graphs:

Definition 5.1 (Co-strongly perfect graph): A graph $G$ is co-strongly perfect if for every induced subgraph $H$ of $G$ there exists $\boldsymbol{\alpha}=\boldsymbol{\alpha}(H) \in\{0,1\}^{|V(H)|}$ such that $\boldsymbol{\alpha}^{T} \mathbf{M}(H)=\mathbf{e}^{T}$. Equivalently, a graph $G$ is co-strongly perfect, if and only if $G$ contains a clique that intersects every maximal independent set in $G$. It follows from the definition, and from the interference

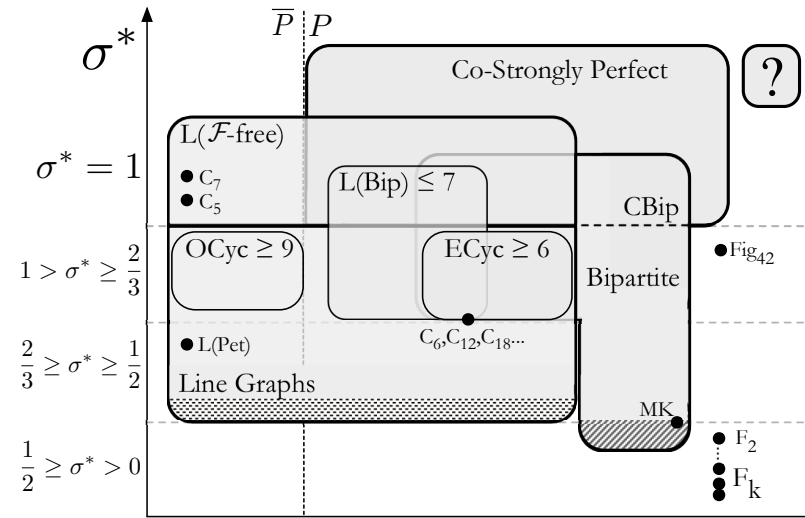

Fig. 4. Throughput guarantees (bounds on $\sigma^{*}$ ) for various interference graph families: $P$ - Perfect graphs, $\bar{P}$ - Non-perfect graphs, ECyc $\geq 6$ - cycles $C_{n}$ with $n$ even and $n \geq 6$, OCyc $\geq 9$ - cycles $C_{n}$ with $n$ odd and $n \geq 9$, $\mathrm{L}$ (Bip) $\leq 7$ - line graphs of $k \times n$ bipartite graphs with $k \leq 7$, CBip Chordal bipartite graphs, L(Pet) - Line graph of the Petersen graph, $\mathrm{L}(\mathcal{F}$-free $)$ - Line graphs of $\mathcal{F}$-free graphs, Fig ${ }_{42}$ - Graph from Fig. 42 in [14], MK Möbius-Kantor graph, $F_{2}$ - graph obtained by a single cycle substitution, $F_{k}$ - sequence of graphs obtained by recursive cycle substitutions.

graph counterparts of Definitions 2.2 and 2.3 that every graph that is co-strongly perfect satisfies OLoP.

Note from the above weighting that co-strongly perfect graphs satisfy OLoP with an integer vector $\boldsymbol{\alpha}$. An open question is whether all perfect graphs that satisfy OLoP do so with integer weights $\alpha$. This is not true for imperfect graphs, because $C_{5}$ is an imperfect graph that satisfies OLoP with the unique optimal node weighting $\boldsymbol{\alpha}(v)=1 / 2$ for all $v \in V\left(C_{5}\right)$. The vertical division of Fig. 4 into perfect and non-perfect graphs, denoted $P$ and $\bar{P}$, respectively, allows us to represent this open problem by the question mark in the perfect division.

The Co-Strongly Perfect class includes a large number of perfect graph families (some of them identified individually in [31]). To provide some context about the magnitude of the result, consider the set of simple graphs with 10 nodes. There are 3,063,185 such co-strongly perfect graphs. This can be compared to the 126,768 chordal graphs with 10 nodes (the chordal graphs family is one of the largest previously known families satisfying OLoP) and to the 106 trees [27].

We proved in Section III-A that $\mathcal{F}$-free network graphs are OLoP-satisfying under primary interference. This is shown in Fig. 4 by the class $\mathrm{L}(\mathrm{F}-\mathrm{f}$ ree) (line graphs of $\mathcal{F}$-free graphs), which is a subclass of the Line Graphs family. Since $\mathrm{L}(\mathcal{F}$-free $)$ graphs represent all OLoP-satisfying line graphs, this family covers the entire section of Line Graphs that is in the $\sigma^{*}=1$ division. The chordal bipartite family, denoted CBip on Fig. 4, is another family that is entirely OLoPsatisfying and forms the subclass of Bipartite graphs that are co-strongly perfect and OLoP-satisfying [31].

\section{B. $\sigma^{*}$-values for Line Graphs}

We examine the $\sigma^{*}$ values of interference graphs that are Line Graphs and that fail OLoP. As mentioned in Section II and in [21], $\sigma^{*} \geq 1 / 2$ for all Line Graphs. In Fig. 4, the bottom part of this family is shaded to indicate that we still 
do not have any specific example of a line graph for which $\sigma^{*}=1 / 2$. The line graph with the lowest known $\sigma^{*}$ value $\left(\sigma^{*}=0.6\right)$ is the line graph of the Petersen graph (Fig. 1-(c)) [16], denoted L (Pet).

We consider families that are subclasses of line graphs. The results on bipartite network graphs from Section IV (line graphs of subgraphs of $K_{t, n}$ with $t \leq 7$ have $\sigma^{*} \geq 2 / 3$ ) are shown on the figure as the $\mathrm{L}(\mathrm{Bip}) \leq 7$ class which is located in the top and the second divisions.

We now obtain the $\sigma^{*}$ values of the entire family of cycles, some of which have been considered individually in the literature. For the 6-cycle it has been shown that $\sigma^{*}=2 / 3$ [9], [16] (represented by the point $\mathrm{C} 6$ on Fig. 4). It has also been shown that C5 and C7 satisfy OLoP, while larger cycles $(n \geq 8)$ do not [31]. Using Lemma 2.3, the following lemma provides the $\sigma^{*}$ of all cycles.

Lemma 5.1: For $n \geq 3, \sigma^{*}\left(C_{n}\right)=\lceil n / 3\rceil /\lfloor n / 2\rfloor$.

Proof: Let $n \geq 3$. Since every proper induced subgraph $H$ of $C_{n}$ (i.e. $H \neq C_{n}$ ) is a forest, we have $\sigma(H)=1$ for every such $H$. Now consider $C_{n}$ itself. A maximum independent set in $C_{n}$ can be constructed by choosing nodes alternatingly on the cycle. This implies that $\bar{\nu}(G)=\lfloor n / 2\rfloor$. A smallest maximal independent set can be constructed by choosing nodes skipping two nodes at a time. This implies that $\bar{\mu}(G)=\lceil n / 3\rceil$. Since $C_{n}$ is vertex-transitive, it follows from Lemma 2.3 that $\sigma(G)=\lceil n / 3\rceil /\lfloor n / 2\rfloor$. From this and the above, the result follows from the definition of $\sigma^{*}(G)$.

To the best of our knowledge, this is the first time an entire family's $\sigma$-value has been characterized this precisely. This result is shown in Fig. 4 as the classes ECYc $\geq 6$ and OCYc $\geq 9$, for large even and odd cycles, respectively. No odd cycle can have $\sigma^{*}=2 / 3$, which is why the OCYC family is strictly within the second division. The family of even cycles is exactly the intersection of the Bipartite and the Line Graphs families that do not satisfy OLoP. In other words, there are no bipartite line graphs that have $\sigma^{*}<1$ and that are not large even cycles. Since $\lceil n / 3\rceil /\lfloor n / 2\rfloor \geq 2 / 3$ for all $n \geq 3$, Lemma 5.1 provides a lower-bound of $2 / 3$ for arbitrary cycles, resulting in the following corollary:

Corollary 5.1: For all cycles, $\sigma^{*}\left(C_{n}\right) \geq 2 / 3$.

\section{Low $\sigma^{*}$-values}

We now focus on graphs with very low $\sigma^{*}$. The current knowledge of $\sigma^{*}$-values is limited to a handful graphs in which GMS achieves a large portion of the stability region. The lowest $\sigma^{*}$-value of a specific graph is $\sigma^{*}=0.6$ for the line graph of the Petersen graph [16]. In [17], it was shown that for geometric graphs $1 / 6 \leq \sigma^{*} \leq 1 / 3$. Below, we present a graph that has $\sigma^{*}=0.5$ and provide a method through which it is possible to create networks with arbitrarily low $\sigma^{*}$.

Consider the graph shown in Fig. 5-(a). It is a generalized Petersen graph with factors $G P(8,3)$, also known as the Möbius-Kantor graph $\mathcal{M K}$. Because of its vertex-transitivity, it follows from Lemma 2.3 and from the fact that $\bar{\nu}(\mathcal{M K})=8$ and $\bar{\mu}(\mathcal{M K})=4$ that $\sigma^{*}(\mathcal{M K})=1 / 2$. Hence, GMS can (a)

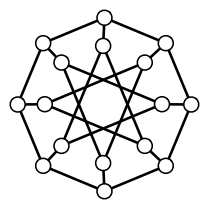

(b)

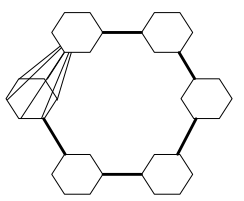

Fig. 5. Graphs that have low $\sigma^{*}$ values: (a) Möbius-Kantor graph (b) $F_{2}$, a graph where each node of a $C_{6}$ is substituted by a $C_{6}$.

only guarantee $50 \%$ throughput. $^{6}$ Being a bipartite graph, the Möbius-Kantor implies that Bipartite graphs can have $\sigma^{*}$ values as low as 0.5 , as illustrated in Fig. 4. Whether bipartite graphs can have $\sigma^{*}<0.5$ is still an open question, shown by the shaded region in Fig. 4.

Now consider the following family. Let $F_{1}$ be a 6-cycle and, for $k \geq 2$, construct $F_{k}$ from $F_{k-1}$ by substituting a 6-cycle for each node $v \in V\left(F_{k}\right)$. By substituting $C_{6}$ for a node $x$ of the original graph, we mean that we replace $x$ by a 6 -cycle $H$ and we make every $v \in V(H)$ adjacent to every neighbor of $x$. For example, $F_{2}$ is shown in Fig. 5-(b), (where the hexagons represent 6-cycles). Using Lemma 2.3 and the fact that the $F_{k}$ are vertex-transitive, we prove the following:

Observation 5.1: $\sigma^{*}\left(F_{k}\right) \leq(2 / 3)^{k}$ for all $k \geq 1$.

Since we may choose $k$ arbitrarily large, it follows that there exist graphs with arbitrarily small $\sigma^{*}$. A graph generated by this method appears in Fig. 4 as F2 and the sequence of graphs obtained through recursive substitution with decreasing $\sigma^{*}$ values is shown as $\mathrm{Fk}$.

Finally, it can be shown that the family of weakly chordal graphs that was left unresolved in [31] is not entirely OLoPsatisfying. An example of a weakly chordal graph that is not co-strongly perfect and that has $\sigma^{*}<1$ appears in Fig. 42 in [14] and is denoted in Fig. 4 as Fig42.

\section{Simulation Results}

When GMS guarantees only low throughput efficiency $\gamma^{*}$, there may exist a specific arrival rate outside of $\gamma^{*} \Lambda^{*}$ for which GMS is not stable. In real-life arrival processes, it is sometimes unlikely that such an arrival process would occur. Hence, GMS may behave better than predicted. We used Matlab simulations in order to evaluate the performance of GMS in graphs with low $\sigma^{*}$ identified in Section V-B.

We consider i.i.d. uniform arrivals to every node at each time slot for a range of normalized loads within the stability region. We tested the GMS and the stable algorithm that solves the Maximum Weight Independent Set problem ${ }^{7}$. For each arrival rate, the simulation was run for 10,000 iterations. For each graph and arrival rate value, the average queue lengths appear in Fig. 6 . The cycle $C_{12}$ has $\sigma^{*}=2 / 3$. In the figure, we see that in a cycle, the queues under GMS become unstable at around load level of 0.85. Although the Möbius-Kantor graph has a $\sigma^{*}=1 / 2$, GMS performs similarly.

\footnotetext{
${ }^{6}$ Note that since this graph contains a claw (i.e., a complete bipartite graph $K_{1,3}$ ), it cannot be the interference graph of any network under primary interference constraints.

${ }^{7}$ Although the problem is NP-complete, we obtained numerical solutions in small graphs.
} 


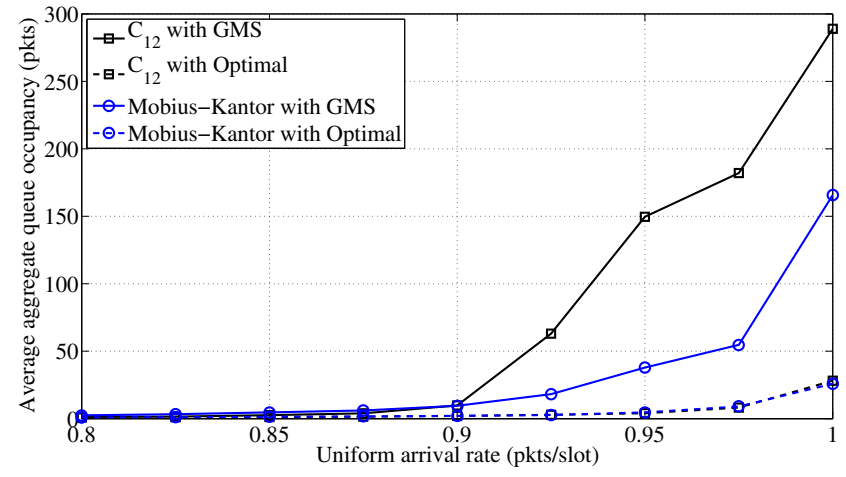

Fig. 6. Average queue sizes as a function of the arrival rate under GMS and the optimal algorithm. The results obtained via simulation in a 12-cycle and a Möbius-Kantor graph.

\section{CONCLUSION}

The Local Pooling (LoP) conditions provide a new tool for better understanding the performance of Greedy Maximal Scheduling (GMS) algorithms. In this paper, we identified all the network graphs in which these conditions hold under primary interference constraints (in these graphs Greedy Maximal Scheduling achieves $100 \%$ throughput). In addition, we showed that in all bipartite graphs of size up to $7 \times n$, GMS is guaranteed to achieve $66 \%$ throughput. Finally, we studied the performance of GMS in interference graphs and showed that $\sigma^{*}$ can be arbitrarily low.

We emphasize that our objective in this paper is to obtain a better theoretical understanding of LoP that will assist the development of future algorithms. As such, the paper demonstrates that using graph theoretical methods can significantly contribute to our understanding of greedy scheduling algorithms. From a graph theoretical point of view, LoP raises many interesting open problems. For example, three of the authors [6], [7] are currently working on extending some of the results to claw-free graphs, which are a generalization of the interference graphs of networks under primary interference. From the networking point of view, there remain many open problems. For example, generalizing the interference model to a model based on SINR and deriving the corresponding LoP conditions remain major subjects for future research.

\section{REFERENCES}

[1] M. Ajmone Marsan, E. Leonardi, M. Mellia, and F. Neri, "On the stability of isolated and interconnected input-queueing switches under multiclass traffic," IEEE Trans. Inform. Th., vol. 51, no. 3, pp. 11671174, Mar. 2005.

[2] B. Birand, M. Chudnovsky, B. Ries, P. Seymour, G. Zussman, and Y. Zwols, "Analyzing the performance of greedy maximal scheduling via local pooling and graph theory," Columbia University, EE, Tech. Rep. \#2009-07-30, July 2009. [Online]. Available: http://www.columbia.edu/ bb2408/pdfs/AnalyzingGMS.pdf

[3] H. Bodlaender, "On linear time minor tests with depth-first search," $J$. Algorithms, vol. 14, no. 1, pp. 1-23, 1993.

[4] A. Brzezinski, G. Zussman, and E. Modiano, "Enabling distributed throughput maximization in wireless mesh networks - a partitioning approach," in Proc. ACM MOBICOM'06, Sept. 2006.
[5] P. Chaporkar, K. Kar, X. Luo, and S. Sarkar, "Throughput and fairness guarantees through maximal scheduling in wireless networks," IEEE Trans. Inform. Th., vol. 54, no. 2, pp. 572-594, Feb. 2008.

[6] M. Chudnovsky, B. Ries, and Y. Zwols, "Claw-free graphs with strongly perfect complements. Fractional and integral version. Part I: Graphs with $\alpha \leq 3$ and fuzzy long circular interval graphs." 2009, in preparation.

[7] — "Claw-free graphs with strongly perfect complements. Fractional and integral version. Part II: Graphs with $\alpha \geq 4$ that are not fuzzy long circular interval graphs." 2009, in preparation.

[8] J. G. Dai and B. Prabhakar, "The throughput of data switches with and without speedup," in Proc. IEEE INFOCOM'O0, Mar. 2000.

[9] A. Dimakis and J. Walrand, "Sufficient conditions for stability of longest queue first scheduling: second order properties using fluid limits," $A d v$. Appl. Probab., vol. 38, no. 2, pp. 505-521, June 2006.

[10] L. Georgiadis, M. J. Neely, and L. Tassiulas, Resource Allocation and Cross-Layer Control in Wireless Networks. NOW Publishers, 2006.

[11] P. Giaccone, B. Prabhakar, and D. Shah, "Randomized scheduling algorithms for high-aggregate bandwidth switches," IEEE J. Select. Areas Commun., vol. 21, no. 4, pp. 546-559, May 2003.

[12] J. Gross and J. Yellen, Graph theory and its applications. CRC press, 2006.

[13] J.-H. Hoepman, "Simple distributed weighted matchings," Oct. 2004 eprint cs.DC/0410047.

[14] S. Hougardy, "Classes of perfect graphs," Discrete Math., vol. 306, no. 19-20, pp. 2529-2571, 2006.

[15] K. Jain, J. Padhye, V. N. Padmanabhan, and L. Qiu, "Impact of interference on multi-hop wireless network performance," ACM/Springer Wireless Networks, vol. 11, no. 4, pp. 471-487, July 2005.

[16] C. Joo, X. Lin, and N. B. Shroff, "Performance limits of greedy maximal matching in multi-hop wireless networks," in Proc. IEEE CDC'07, Dec. 2007.

[17] C. Joo, X. Lin, and N. Shroff, "Understanding the capacity region of the greedy maximal scheduling algorithm in multi-hop wireless networks," in Proc. IEEE INFOCOM'08, April 2008.

[18] F. Kuhn, T. Moscibroda, and R. Wattenhofer, "What cannot be computed locally!" in Proc. ACM PODC'04, July 2004.

[19] M. Leconte, J. Ni, and R. Srikant, "Improved bounds on the throughput efficiency of greedy maximal scheduling in wireless networks," in Proc. ACM MOBIHOC'09, May 2009.

[20] B. Li, C. Boyaci, and Y. Xia, "A refined performance characterization of longest-queue-first policy in wireless networks," in Proc. ACM MOBIHOC'09, May 2009.

[21] X. Lin and N. B. Shroff, "The impact of imperfect scheduling on cross-layer rate control in wireless networks," IEEE/ACM Trans. Netw., vol. 14, no. 2, pp. 302-315, Apr. 2006.

[22] F. Maffray, "Kernels in perfect line-graphs," J. Combin. Theory Ser. B, vol. 55, no. 1, pp. 1-8, 1992.

[23] N. McKeown, A. Mekkittikul, V. Anantharam, and J. Walrand, "Achieving $100 \%$ throughput in an input-queued switch," IEEE Trans. Commun., vol. 47, no. 8, pp. 1260-1267, Aug. 1999.

[24] T. Moscibroda and R. Wattenhofer, "The complexity of connectivity in wireless networks," in Proc. IEEE INFOCOM'06, Apr. 2006.

[25] R. Preis, "Linear time 1/2-approximation algorithm for maximum weighted matching in general graphs," in $L N C S$, vol. 1563, pp. 259269, 1999.

[26] G. Sharma, R. R. Mazumdar, and N. B. Shroff, "On the complexity of scheduling in wireless networks," in Proc. ACM MOBICOM'06, Sept. 2006.

[27] N. J. A. Sloane. (2009) The on-line encyclopedia of integer sequences. [Online]. Available: http://www.research.att.com/ njas/sequences/

[28] L. Tassiulas and A. Ephremides, "Stability properties of constrained queueing systems and scheduling policies for maximum throughput in multihop radio networks," IEEE Trans. Automat. Contr., vol. 37, no. 12, pp. 1936-1948, Dec. 1992.

[29] D. West, Introduction to Graph Theory. Prentice Hall, Upper Saddle River, NJ, 2001

[30] X. Wu and R. Srikant, "Bounds on the capacity region of multi-hop wireless networks under distributed greedy scheduling," in Proc. IEEE INFOCOM'06, Apr. 2006

[31] G. Zussman, A. Brzezinski, and E. Modiano, "Multihop local pooling for distributed throughput maximization in wireless networks," in Proc. IEEE INFOCOM'08, April 2008 